\title{
Studien über die umwallten Zungenpapillen bei den japanischen Zwillingsfeten.
}

\author{
Von \\ Hisao Ogawa. \\ Aus dem Anatomischen Institut der Keio Universität, Tokyo. \\ (Direktor: Prof. Dr. T. Taniguchi). \\ Mit 22 Textfiguren.
}

\section{Einleitung.}

Morphologische und anthropologische Untersuchungen über die Papillae vallatae sind seit jeher von vielen Autoren, wie Giacomini (1884), Münch (1886), Stöhr (I902), Hopf (1910), Edzard (I9ro), Grabert (1910), Kunitomo (1910), Zuckermann (1931), Jurisch (1922), Hishi (1927), Kukita- (1932), Miyashita (1935), Mochizuki (1938), u.a: angestellt worden. Die Untersuchungssphäre erstreckt sich auf 8 Rassengruppen, d.h. Neubritanier (Hopf, Edzard), Herero (Grabert), Hottentotte (Grabert), Melanesier (Zuckermann), Europäer (Grabert, Jurisch), Chinese (Miyashita), Ainu (Kukita) und Japaner (Kunitomo, Mochizuki, Nishi), deren eingehende Forschung sich weiter auf Simiidae oder Anthropoidae (Kunze) ausgedehnt hat. So findet man über die Papillae vallatae sehr umfangreiche, genaue Beobachtungen, die dazu beigetragen haben, unsere Kenntnisse weitgehenst zu bereichern. Wir finden aber keine derartigen Untersuchungen über Zwillingsfeten.

$\mathrm{Da}$ ich die vorliegende Untersuchung untenommen habe, um diese Lücke gewissermassen-auszufüllen und dadurch zur Kenntnis der Erbbiologie beizutragen, will ich mein Resultat im folgenden kurz mitteilen.

\section{Material und Methode.}

Als Untersuchungsmaterial wählte ich von 70 paärigen Zwillingsfeten, welche von Prof. Dr. T. Ta ni g u chi in unserem Institut für seine morphologischen und vergleichend anatomischen Untersuchungen reserviert sind, 
2I paarige 7-9 monatige Zwillinge, bei denen ich die Papillea vallatae makroskopisch untersuehen konnte.

Sie setzen sich aus 14 EZ-Paaren (송 Io, 우우 4), 7 ZZ-Paaren (양 2,

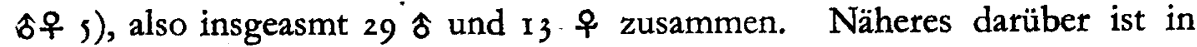
der Tabelle I zusammengestellt.

Die meisten Leichen wurden mit 10\% Formolalkohol im ganzen Körper injiziert und in 10\% Formollösung konserviert.

Tabelle I. Untersuchungsmaterial.

\begin{tabular}{|c|c|c|c|c|c|c|}
\hline Fall & Eiigkeit & $\begin{array}{l}\text { Embryo- } \\
\text { nummer }\end{array}$ & Geschlecht & Rumpflänge & $\begin{array}{l}\text { Körper- } \\
\text { gewicht }\end{array}$ & Fetalmonat \\
\hline I & $\mathrm{EZ}$ & $\begin{array}{l}566 \\
567\end{array}$ & $\boldsymbol{\delta}$ & $\begin{array}{l}13.7 \mathrm{~cm} \\
13.5\end{array}$ & $\begin{array}{l}2070 \mathrm{gr} \\
1830\end{array}$ & 9 \\
\hline 2 & $\mathrm{EZ}$ & $\begin{array}{l}768 \\
770 \\
\end{array}$ & 우 & $\begin{array}{r}12.7 \\
13.6 \\
\end{array}$ & $\begin{array}{l}1 \times 50 \\
1320 \\
\end{array}$ & 8 \\
\hline 3 & $\mathrm{EZ}$ & $\begin{array}{l}1057 \\
1058 \\
\end{array}$ & 全 & $\begin{array}{l}12.0 \\
12.5 \\
\end{array}$ & $\begin{array}{l}1040 \\
1100 \\
\end{array}$ & 8 \\
\hline 4 & $\mathrm{EZ}$ & $\begin{array}{l}1107 \\
1108 \\
\end{array}$ & ธิ & $\begin{array}{l}15.0 \\
15.0\end{array}$ & $\begin{array}{l}1875 \\
1650\end{array}$ & 9 \\
\hline 5 & $\overline{E Z}$ & $\begin{array}{l}1254 \\
1255\end{array}$ & ิิ & $\begin{array}{l}14.7 \\
15.0 \\
\end{array}$ & $\begin{array}{r}1470 \\
1635 \\
\end{array}$ & 8 \\
\hline 6 & $\mathrm{EZ}$ & $\begin{array}{l}1261 \\
1262 \\
\end{array}$ & 우 & $\begin{array}{l}\mathrm{I} 2.2 \\
\mathrm{I} 1.8 \\
\end{array}$ & $\begin{array}{r}1030 \\
930 \\
\end{array}$ & 7 \\
\hline 7. & $\mathrm{EZ}$ & $\begin{array}{l}1300 \\
1301 \\
\end{array}$ & $\delta$ & $\begin{array}{l}14.0 \\
14.0 \\
\end{array}$ & $\begin{array}{r}1580 \\
1330 \\
\end{array}$ & 9 \\
\hline 8 & $\mathrm{EZ}$ & $\begin{array}{l}1424 \\
1425\end{array}$ & 占 & $\begin{array}{l}10.5 \\
10.5\end{array}$ & $\begin{array}{l}720 \\
630\end{array}$ & 7 \\
\hline 9 & EZ & $\begin{array}{l}1431 \\
1432\end{array}$ & 우 & $\begin{array}{l}13.8 \\
13.0 \\
\end{array}$ & $\begin{array}{r}1250 \\
995 \\
\end{array}$ & 7 \\
\hline Io & $\mathrm{EZ}$ & $\begin{array}{l}1445 \\
1446\end{array}$ & $\boldsymbol{\delta}$ & $\begin{array}{l}13.0 \\
13.5\end{array}$ & $\begin{array}{l}I 120 \\
1560\end{array}$ & 9 \\
\hline II & EZ & $\begin{array}{l}1453 \\
1454\end{array}$ & o & $\begin{array}{l}14.0 \\
12.5\end{array}$ & $\begin{array}{l}1140 \\
1070\end{array}$ & 8 \\
\hline 12 & $\mathrm{EZ}$ & $\begin{array}{l}1514 \\
1515 \\
\end{array}$ & $\boldsymbol{\delta}$ & $\begin{array}{l}13.5 \\
13.5 \\
\end{array}$ & $\begin{array}{l}1475 \\
1290 \\
\end{array}$ & 8 \\
\hline 13 & $\mathrm{EZ}$ & $\begin{array}{r}1537 \\
1538 \\
\end{array}$ & 18 & $\begin{array}{r}14.0 \\
13.5 \\
\end{array}$ & $\begin{array}{l}1325 \\
1195 \\
\end{array}$ & 9 \\
\hline 14 & $\mathrm{EZ}$ & $\begin{array}{l}1551 \\
1552\end{array}$ & 8 & $\begin{array}{r}12.8 \\
\mathbf{1} 3.0 \\
\end{array}$ & $\begin{array}{r}1290 \\
1350\end{array}$ & 8 \\
\hline is & $\mathrm{ZZ}$ & $\begin{array}{l}762 \\
763\end{array}$ & 웅 & $\begin{array}{l}12.0 \\
12.0\end{array}$ & $\begin{array}{r}1020 \\
94^{\circ} \\
\end{array}$ & 8 \\
\hline 16 & $\mathrm{ZZ}$ & $\begin{array}{l}765 \\
769\end{array}$ & $\begin{array}{l}\text { S } \\
\text { \& }\end{array}$ & $\begin{array}{l}16.2 \\
18.1\end{array}$ & $\begin{array}{l}2200 \\
2600\end{array}$ & 9 \\
\hline 17 & $\mathrm{ZZ}$ & $\begin{array}{l}961 \\
962 \\
\end{array}$ & $\begin{array}{l}\hat{\delta} \\
\hat{\delta}\end{array}$ & $\begin{array}{r}13.0 \\
13.0 \\
\end{array}$ & $\begin{array}{r}1125 \\
980 \\
\end{array}$ & 7 \\
\hline 18 & $\mathrm{ZZ}$ & $\begin{array}{l}1080 \\
1081\end{array}$ & $\begin{array}{l}\hat{8} \\
8\end{array}$ & $\begin{array}{r}14.0 \\
14.5 \\
\end{array}$ & $\begin{array}{r}1235 \\
1140 \\
\end{array}$ & 7 \\
\hline 19 & $\mathrm{ZZ}$ & $\begin{array}{l}1323 \\
1324 \\
\end{array}$ & $\hat{\delta}$ & $\begin{array}{l}13.3 \\
15.0 \\
\end{array}$ & $\begin{array}{l}1430 \\
1200- \\
\end{array}$ & 9 . \\
\hline 20 & $\mathrm{ZZ}$ & $\begin{array}{l}1532 \\
1539\end{array}$ & क & $\begin{array}{r}14.0 \\
\times 2.5\end{array}$ & $\begin{array}{r}\text { IIIS } \\
880 \\
\end{array}$ & 7 \\
\hline $2 \mathrm{I}$ & $\mathrm{ZZ}$ & $\begin{array}{r}1558 \\
1559 \\
\end{array}$ & 웅 & $\begin{array}{l}10.2 \\
12.0 \\
\end{array}$ & $\begin{array}{r}800 \\
1130 \\
\end{array}$ & 7 \\
\hline
\end{tabular}




\section{Eigene Befunde.}

Bevor ich auf meine eigenen Untersuchungen eingehe, will ich zuerst für die Namen der einzelnen Wallpapillen die Münch'sche Normenklatur zugrunde legen, um ihre Zahl, Grösse und Stellung genügend zu erklären.

(1)

(2)

(3) (8)

(7)

(10)

(11)

Fig. I. Nummer der einzelnen Wallpapillen

nach $M \ddot{u} n c h$.
I. die vordere
2. die hintere Papilla vallata accessoria lateralis sinistra,

4. die vordere
s. die hintere Papilla vallata accessoria lateralis dextra,

3. Papilla vallata lateralis sinistra,

6. Papilla vallata lateralis dextra,

7. Papilla vallata centralis,

8. die erste 3 Papilla vallata mediana anterior,

I0. die erste
Ix. die zweite Papilla vallata mediana posterior.

Aber in der vorliegenden Untersuchung werden die Papillae vallatae nur in die laterale und mediane Papillenreihe unterschieden, um die Zahl und Stellung der Papillae vallatae zu erklären.

Näheres über die eigenen Befunden ist in der Tabelle 2 zusammengestellt.

\section{Zusammenfassung.}

Ich habe mich hauptsächlich mit der Stellung, dem Winkel der beiderseitigen Schenkel, mit der Zahl der Papillae vallatae bei den Zwillingsfeten beschäftigt, soweit die makroskopische Untersuchung der Papillae vallatae in Betracht. kommt.

\section{r) Die Stellung der Papillae vallatae.}

Bekanntlich ist die Stellung der Papillae vallatae an der Zunge beim Menschen in die folgenden 3 Typen unterschieden worden :

I. die V-Stellung,

2. die Y-Stellung,

3. die T-Stellung.

Benannt habe ich als die T-Stellung eine solche, bei welcher der Winkel der lateralen Papillen so gross ist, dass er über $150^{\circ}$ beträgt, was schon von den vielen Autoren erwähnt ist. Keinmal habe ich die Figur angetroffen, 


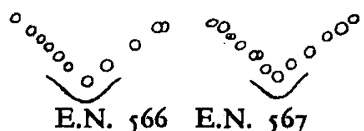

E.N. 566 E.N. 567
Fig. 2, Fall I, EZ.

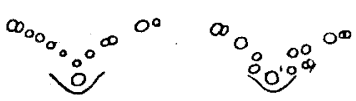

E.N. 1057 E.N. 1058

Fig. 4, Fall 3, EZ.

$0_{0} 0_{0} 0^{\circ} 0_{0} 0^{\circ}$

E.N. 1254 E.N. 1255
Fig. 6, Fall 5, EZ.

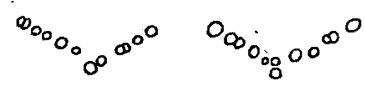

E.N. 1300 E.N. 1301

Fig. 8, Fall 7, EZ.

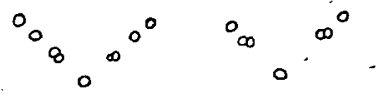

E.N. 1431 E.N. 1432

Fig. 10, Fall 9; EZ.

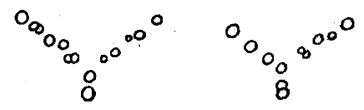

E.N. 1453 E.N. 1454

Fig. 12, Fall II, EZ.

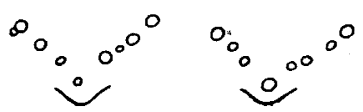

E.N. 1537 E.N. 1538

Fig. 14, Fall 13, EZ.

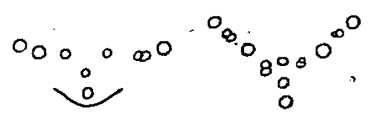

E.N. $76_{2} \quad$ E.N. $76_{3}$

Fig. 16, Fall Is, ZZ.

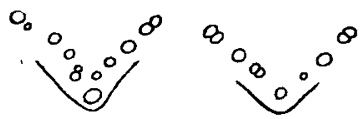

E.N. $96 \mathrm{I}$ E.N. 962

Fig. 18, Fall 17, $\mathrm{ZZ}$.

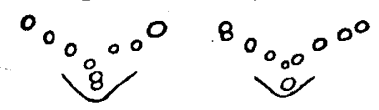

E.N. ${ }^{1323}$ E.N. ${ }^{1324}$
Fig. 20, Fall $19, \quad Z Z$.

$$
\begin{array}{cc}
0_{000} 0^{\circ} & 0
\end{array}
$$

E.N. I558 E.N. I559
Fig. 22, Fall 21, ZZ.
E.N. 768 E.N. 770

Fig. 3, Fall 2, EZ.

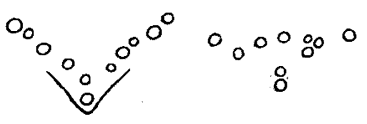

E.N. 1107 E.N. 1108

Fig. 5, Fall 4, EZ.

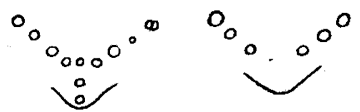

E.N. $x 26 \mathrm{I}$ E.N. 1262

Fig. 7, Fall 6, EZ.

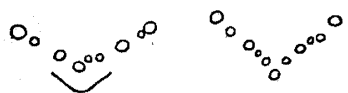

E.N. 1424 E.N. 1425

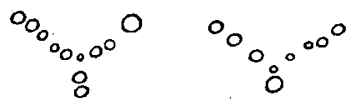

E.N. 1445 E.N. 1446

Fig. Ir, Fall ro, EZ.

$$
0_{0}^{\circ} 0^{\circ} 0^{\circ} \quad 0_{000}
$$

E.N. ISI4 E.N. IIIS

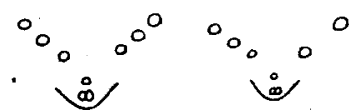

E.N. 1551 E.N. 1552

Fig. 15, Fall I4, EZ.

$$
0_{0}^{\infty} 0^{\circ} 0^{\infty} 0^{0}
$$

E.N. $7^{6} 5$ E.N. $7^{69}$

Fig: 17, Fall 16, ZZ.

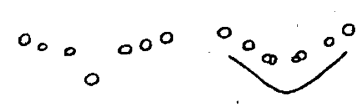

E.N. 1080 E.N. 1081

Fig. 19, Fall $18, \mathrm{ZZ}$.

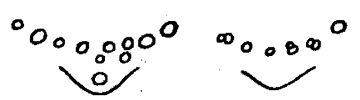

E.N. 1532 E.N. 1539

Fig. 21, Fall 20, ZZ. 
Tabelle 2. Eigene Befunde.

\begin{tabular}{|c|c|c|c|c|c|c|c|c|c|c|}
\hline & & Mate & & & & & & & & \\
\hline 苛 & & ర藍 & 명 & 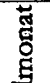 & & & 氠 & घี & ⿷匚⿱ & 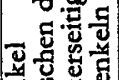 \\
\hline 浔 & 层 & 氠 & రુ & $\begin{array}{c}\text { 苟 } \\
\text { H }\end{array}$ & $\mathbf{R}$ & $\mathbf{L}$ & छั & मีं & . & $\bar{s}$ \\
\hline & I & $\begin{array}{l}566 \\
567\end{array}$ & 今 & 9 & $\begin{array}{l}4(3) \\
5\end{array}$ & $\begin{array}{l}6 \\
8(6) \\
\end{array}$ & $\begin{array}{l} \\
I\end{array}$ & $\begin{array}{l}\text { II (I0) } \\
\text { I4 (I 2) }\end{array}$ & $\begin{array}{l}\mathrm{V} \\
\mathrm{V}\end{array}$ & $\begin{array}{l}102 \\
102\end{array}$ \\
\hline & 2 & $\begin{array}{l}768 \\
770\end{array}$ & 우 & 8 & $\begin{array}{l}3 \\
3 \\
\end{array}$ & 3. & 2 & $\begin{array}{l}6 \\
8\end{array}$ & $\begin{array}{l}\mathbf{V} \\
Y\end{array}$ & $\begin{array}{l}127 \\
128\end{array}$ \\
\hline & 3 & $\begin{array}{l}1057 \\
1058 \\
\end{array}$ & $\delta$ & 8 & $\begin{array}{l}5(4) \\
8(6)\end{array}$ & $\begin{array}{l}6(5) \\
5(4)\end{array}$ & $\begin{array}{l}2 \\
1\end{array}$ & $\begin{array}{l}\text { I3 (I I) } \\
\text { I0 (8) }\end{array}$ & $\begin{array}{l}\mathrm{Y} \\
\mathrm{V}\end{array}$ & $\begin{array}{l}126 \\
125 \\
\end{array}$ \\
\hline & 4 & $\begin{array}{l}1107 \\
1108 \\
\end{array}$ & 今 & 9 & $\begin{array}{l}5 \\
4\end{array}$ & $\begin{array}{r}4 \\
3 \\
\end{array}$ & $\begin{array}{l}2 \\
3 \\
\end{array}$ & $\begin{array}{l}\text { II } \\
\text { IO }\end{array}$ & $\begin{array}{l}Y \\
V \\
V\end{array}$ & $\begin{array}{l}105 \\
114\end{array}$ \\
\hline & 5 & $\begin{array}{l}1254 \\
1255 \\
\end{array}$ & 今 & 8 & $\begin{array}{l}4 \\
3\end{array}$ & $\begin{array}{l}4 \\
3 \\
\end{array}$ & I & $\begin{array}{l}9 \\
8\end{array}$ & $\begin{array}{l}Y \\
Y\end{array}$ & $\begin{array}{r}130 \\
122 \\
\end{array}$ \\
\hline & 6 & $\begin{array}{l}1261 \\
1262 \\
\end{array}$ & 우 & 7 & $\begin{array}{l}5(4) \\
3\end{array}$ & $\begin{array}{l}4 \\
3 \\
\end{array}$ & 3 & $\begin{array}{r}12 \\
6\end{array}$ & $\begin{array}{l}\mathbf{Y} \\
\mathrm{V}\end{array}$ & $\begin{array}{l}105 \\
102 \\
\end{array}$ \\
\hline & 7 & $\begin{array}{l}1300 \\
1301 \\
\end{array}$ & $\delta$ & 9 & $\begin{array}{l}5(4) \\
5(4)\end{array}$ & $\begin{array}{l}6(5) \\
5(4)\end{array}$ & $\begin{array}{l}1 \\
2\end{array}$ & $\begin{array}{l}12(10) \\
12(10)\end{array}$ & $\begin{array}{l}\mathrm{V} \\
\mathrm{V}\end{array}$ & $\begin{array}{r}120 \\
120 \\
\end{array}$ \\
\hline [工工 & 8 & $\begin{array}{l}1424 \\
1425 \\
\end{array}$ & s & 7 & 5 & $\begin{array}{l}3 \\
5 \\
\end{array}$ & $\begin{array}{r}I \\
-I \\
\end{array}$ & $\begin{array}{r}9 \\
\text { II }\end{array}$ & $\begin{array}{l}\mathrm{V} \\
\mathrm{V}\end{array}$ & $\begin{array}{l}\text { IIS } \\
\text { IOS }\end{array}$ \\
\hline & 9 & $\begin{array}{l}1431 \\
1432 \\
\end{array}$ & 우 & 7 & $\begin{array}{l}4(3) \\
3(2)\end{array}$ & $\begin{array}{l}4(3) \\
3(2)\end{array}$ & $\begin{array}{l}\text { I } \\
\text { I }\end{array}$ & $\begin{array}{l}9(7) \\
7(5)\end{array}$ & $\begin{array}{l}\mathrm{V} \\
\mathrm{V}\end{array}$ & $\begin{array}{r}102 \\
98 \\
\end{array}$ \\
\hline & 10 & $\begin{array}{r}1445 \\
1446 \\
\end{array}$ & $\delta$ & 9 & $\begin{array}{l}3 \\
4\end{array}$ & $\begin{array}{l}5 \\
3\end{array}$ & $\begin{array}{l}3 \\
2\end{array}$ & $\begin{array}{r}\text { II } \\
9\end{array}$ & $\begin{array}{l}Y \\
Y\end{array}$ & $\begin{array}{l}\text { IOS } \\
\text { IIS }\end{array}$ \\
\hline & II & $\begin{array}{r}1453 \\
-1454 \\
\end{array}$ & $\delta$. & 8 & $5(4)$ & $\begin{array}{l}7(6) \\
3\end{array}$ & $\begin{array}{l}2 \\
3(2)\end{array}$ & $\begin{array}{l}\text { I4 (13) } \\
\text { I I (9) }\end{array}$ & $\begin{array}{l}\mathrm{Y} \\
\mathrm{Y}\end{array}$ & $\begin{array}{l}\text { IIO } \\
\text { II } 3\end{array}$ \\
\hline & 12 & $\begin{array}{l}1514 \\
1515\end{array}$ & $\boldsymbol{\delta}$ & 8 & $5(4)$ & $\begin{array}{l}4 \\
4 \\
\end{array}$ & I & $\begin{array}{l}8 \\
9(8)\end{array}$ & $\begin{array}{l}\mathrm{V} \\
\mathrm{V}\end{array}$ & $\begin{array}{l}\text { II3 } \\
\text { II } \\
\end{array}$ \\
\hline & 13 & $\begin{array}{l}1537 \\
I 538\end{array}$ & $\delta$ & 9 & $\begin{array}{r}4 \\
4 \\
\end{array}$ & $\begin{array}{l}4(3)- \\
3\end{array}$ & $\begin{array}{l} \\
I\end{array}$ & $\begin{array}{l}9(8) \\
8\end{array}$ & $\begin{array}{l}\mathrm{V} \\
\mathrm{V}\end{array}$ & $\begin{array}{l}100 \\
100\end{array}$ \\
\hline & 14 & $\begin{array}{l}1551 \\
155,2\end{array}$ & 우 & 8 & $\begin{array}{l}3 \\
2\end{array}$ & $\begin{array}{l}3 \\
3\end{array}$ & $\begin{array}{l}3(2) \\
3(2)\end{array}$ & $\begin{array}{l}9(8) \\
8(7)\end{array}$ & $\begin{array}{l}Y \\
Y\end{array}$ & $\begin{array}{r}96 \\
103\end{array}$ \\
\hline & 15 & $\begin{array}{l}762 \\
763 \\
\end{array}$ & $\frac{9}{8}$ & 8 & $\begin{array}{l}4(3) \\
6(4) \\
\end{array}$ & $\begin{array}{l}3 \\
6(4)\end{array}$ & $\begin{array}{l}2 \\
3\end{array}$ & $\begin{array}{c}9(8) \\
15(\mathrm{II})\end{array}$ & $\begin{array}{l}\mathrm{T} \\
\mathrm{Y}\end{array}$ & $\begin{array}{l}155 \\
100\end{array}$ \\
\hline & $x 6$ & $\begin{array}{l}765 \\
769 \\
\end{array}$ & $\begin{array}{l}\hat{\delta} \\
\text { q }\end{array}$ & 9 & $\begin{array}{l}5(3) \\
4(3)\end{array}$ & $\begin{array}{l}5(4) \\
4\end{array}$ & $\begin{array}{l}2(\mathrm{I}) \\
\mathrm{I}\end{array}$ & $\begin{array}{r}12(8) \\
9(8) \\
\end{array}$ & $\begin{array}{l}\mathrm{Y} \\
\mathrm{V}\end{array}$ & $\begin{array}{l}\text { 115 } \\
107\end{array}$ \\
\hline & 17 & $\begin{array}{l}961 \\
962 \\
\end{array}$ & 官 & 7 & $\begin{array}{l}5 \\
4(3)\end{array}$ & $\begin{array}{l}6(5) \\
5(3)\end{array}$ & I & $\begin{array}{l}\text { I } 2 \text { (II) } \\
\text { Xo (7) }\end{array}$ & $\begin{array}{l}\mathrm{V} \\
\mathrm{V}\end{array}$ & $\begin{array}{r}92 \\
100\end{array}$ \\
\hline $\mathrm{N}$ & 18 & $\begin{array}{l}1080 \\
108 x \\
\end{array}$ & $\begin{array}{l}\text { f } \\
\text { \& }\end{array}$ & 7 & $\begin{array}{l}3 \\
4(3)\end{array}$ & $\begin{array}{l}3 \\
4(3)\end{array}$ & I & $\begin{array}{l}7 \\
8(6)\end{array}$ & $\begin{array}{l}Y \\
V\end{array}$ & $\begin{array}{l}140 \\
123\end{array}$ \\
\hline & 19 & $\begin{array}{l}1323 \\
1324 \\
\end{array}$ & 定 & 9 & $\begin{array}{l}3 \\
4\end{array}$ & $\begin{array}{l}4 \\
4(3)\end{array}$ & $\begin{array}{l}2(1) \\
2\end{array}$ & $\begin{array}{r}9(8) \\
\text { ro( }\end{array}$ & $\begin{array}{l}\mathbf{Y} \\
\mathbf{Y}\end{array}$ & $\begin{array}{l}\text { II } 4 \\
120\end{array}$ \\
\hline & 20 & $\begin{array}{l}1532 \\
1539\end{array}$ & 우 & 7 & $5(3)$ & $\begin{array}{l}4 \\
3\end{array}(2)$ & $\begin{array}{l}2 \\
1\end{array}$ & $\begin{array}{l}\text { II } \\
9(6)\end{array}$ & $\begin{array}{l}\mathbf{Y} \\
\mathrm{V}\end{array}$ & $\begin{array}{l}140 \\
145\end{array}$ \\
\hline & $2 I$ & $\begin{array}{l}1558 \\
1559\end{array}$ & 우 & 7 & $\begin{array}{l}3 \\
4(3)\end{array}$ & $\begin{array}{l}5(4) \\
4\end{array}$ & $\begin{array}{l}\text { I } \\
\text { I }\end{array}$ & $\begin{array}{l}9(8) \\
9(8)\end{array}$ & $\begin{array}{l}\mathrm{V} \\
\mathrm{Y}\end{array}$ & $\begin{array}{l}112 \\
145\end{array}$ \\
\hline
\end{tabular}

Die Ziffer in Klammer zeigt die Zahl der Umwallungen.

welcher die medianen Papillen von T-Stellung fehlen und welche sich durch eine gerade Linie kennzeichnet, wie Grabert sie beim Afrikaner fand.

Ich habe zu der Y-Stellung auch die V-förmige Stellung eingerechnet, deren Zentralpapille nicht am Gipfel des von den lateralen Papillen gebildeten 
Winkels steht, sondern sich davon nach hinten schiebt und hinter derselben sich keine Papillen mehr befinden, damit sie von der typischen V-Stellung unterschieden werden kann, an der die zentrale gerade am Scheitel steht. Manchmal kommt nur eine mediane Papille nicht nur in der Y-Stellung vor, sondern auch in der T-Stellung.

Durch oben erwähnte Einteilung möchte ich nun im nachstehenden zur Untersuchung der Stellung der Papillae vallatae bei den Zwillingen übergehen.

- Tabelle 3. Häufigkeit der verschiedenen Stellung der Papillae vallatae an den einzelnen Zungen.

\begin{tabular}{|c|c|c|c|c|c|c|c|c|c|}
\hline \multirow{2}{*}{ Stellung } & \multicolumn{2}{|c|}{ nach der Eiigkeit } & \multicolumn{3}{|c|}{$\begin{array}{l}\text { nach dem } \\
\text { Geschlecht }\end{array}$} & \multicolumn{3}{|c|}{ nach dem Fetalmonat } & \multirow{2}{*}{ Summe } \\
\hline & $\begin{array}{r}\mathrm{EZ} \\
(\mathbf{2 8}) \\
\end{array}$ & $\begin{array}{l}\mathrm{ZZ} \\
(14) \\
\end{array}$ & $\begin{array}{c}\mathbf{8} \\
(29)\end{array}$ & $\begin{array}{c}9 \\
(13)\end{array}$ & & $\begin{array}{c}7 \\
(14) \\
\end{array}$ & $\begin{array}{c}8 \\
(14) \\
\end{array}$ & $\begin{array}{c}9 \\
(14) \\
\end{array}$ & \\
\hline $\mathrm{V}$ & 16 & 6 & IS & 7 & & 10 & 4 & 8 & 22 \\
\hline $\mathbf{Y}$ & 12 & 7 & 14 & 5 & - & 4 & 9 & 6 & $x 9$ \\
\hline$T$ & - & $y$ & - & I & & - & $\mathbf{I}$ & - & I \\
\hline
\end{tabular}

An 42 Zungen der Zwillingsfeten, wie in der Tabelle 3 gezeigt, gehört die Stellung der Papillae vallatae 22 mal zur V-Stellung, I9 mal zur Y-Stelung und I mal zur T-Stellung. Die Häufigkeit der V-und Y-Stellung der Papillae vallatae zeigt keinen bedeutenden Unterschied zwischen EZ und ZZ wie auch zwischen den männlichen Feten und den weiblichen.

$\mathrm{Kunit}$ o mo fand an 20 Zungen bei den japanischen Kindern, dass sich $6 \mathrm{mal}$ die V-Stellung und $4 \mathrm{mal}$ die Y-Stellung zeigt und Moch i $\mathrm{zuki}$ fand an 14 Kindern von I-2 Jahren, dass die Anordnung der Papillae vallatae $s$ mal zur V-Stellung und 9 mal zur Y-Stellung gehört.

Bei den Zwillingsfeten ist die Häufigkeit der V-Stellung grösser als die der Y-Stellung. Diese Tatsache scheint darauf $\mathrm{zu}$ beruhen, dass die Ausbildung der Papillae vallatae medianae posteriores beim Embryo sehr schwach ist.

Nach dem Fetalmonat scheint die Häufigkeit der verschiedenen Stellung einen gewissermassen Unterschied zu zeigen. Aber es bedarf noch genauere statistische Beobachtung an grösseren Materialien, diesen Unterschied an den Tag zu bringen.

Vergleicht man bezüglich der Stellung der Papillae vallatae beide Feten desselben Paares miteinander, so erhält man das Ergebnis wie in Tabelle 4.

Wie aus Tabelle 4 ersichtlich, in bezug auf die Stellung der Papillae vallatae ist der Ähnlichkeitsgrad zwischen zwei Feten desselben Paares bei EZ grösser als bei ZZ. Sonach kann man die Behauptung aussprechen, 
Tabelle 4. Vergleich der Stellung der Papillae vallatae an den einzelnen Zwillingspaaren.

\begin{tabular}{|l|c|c|}
\hline & EZ & ZZ \\
& $(14)$ & $(7)$ \\
\hline Konkordanz & 10 & 2 \\
Diskordanz & 4 & 5 \\
\hline
\end{tabular}

dass die Erbwirkungen auf die Anordnung der Papillae vallatae deutlich nachzuweisen sind.

An einem Partner eines EZ-Paares (E.N. Ios 8) zeigt die Anordnung der Papillae vallatae die mehrreihige VStellung, bei welcher die lateralen Papillen in 2 Reihen angelegt sind.

\section{2) Der Winkel.}

Wie die Kunitomo's Angabe bietet die Methode, die Grösse des Windels möglichst genau zu bestimmen, welchen die beiderseitigen Schenkel der lateralen Papillen bilden, sehr oft Schwierigkeiten dadurch, dass nicht alle lateralen Papillen sich in einer regelmässigen, geraden Reihe anordnen, sondern gewissermassen voneinander abweichend sind, infolgedessen der Scheitelpunkt des Winkels schwer zu bestimmen ist.

Oppel wies nach, dass dieser Winkel sowohl bei Erwachsenen als auch bei Kindern gleich gross ist und dass also beim Wachstum der Zunge weder ein Spitzer- noch ein Stumpfwerden des Winkels eintritt (zit. nach Kunitomo). Kunitomo gibt in seiner Abhandlung an, dass die Winkelgrösse sich beim Wachstum der Zunge nicht wesentlich verändert, obwohl der Winkel an den Kinderzungen etwas grösser als an den der Erwachsenen zu sein scheint. Dagegen ist von Mochizuki jedoch hervorgehoben, dass der Winkel bei den jüngeren Japanern grösser als bei den älteren ist.

Die Mehrzahl der Zungen bei den Zwillingsfeten zeigen einen Winkel von $100^{\circ}-110^{\circ}$; häufig sind auch noch Zungen mit Winkeln von $115^{\circ}$ und

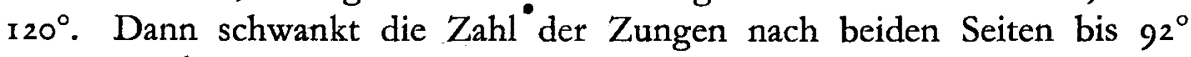

Tabelle s. Vergleich der Winkelgrösse, welche zwischen den beiderseitigen Schenkeln der lateralen

Papillen gebildet wird, an den einzelnen Zwillingspaaren.

\begin{tabular}{|l|c|c|}
\hline & EZ & ZZ \\
\hline (14) & $(7)$ \\
\hline $\begin{array}{c}\text { gleich } \\
\text { fast gleich } \\
\text { (Der Unterschied } \\
\text { ist kleiner als 10 } \\
\text { nicht gleich }\end{array}$ & 3 & - \\
\hline
\end{tabular}
bzw. $155^{\circ}$ ab. Der Winkel zeigt keinen Unterschied nach der Eiigkeit, dem Geschlecht und Alter.

Wenn man bezüglich der Winkelgrösse beide Feten, -desselben Paares miteinander vergleicht, so zeigt sich das Ergebnis wie in Tabelle 5.

Wie aus der Tabelle $s$ zu sehen ist, ist der Ähnlichkeitsgrad der Winkelgrösse bei $\mathrm{EZ}$ deutlich grösser als bei ZZ. Man kann jetzt das wichtige Ergèbnis obiger Befunde über 
den Winkel, welchen die beiderseitigen Schenkel der lateralen Papillen bilden, folgendermassen zusammenfassen, dass die Grösse des Winkels von der Erbwirkung auffallend abhängig ist, ebenso die Anordnung der Papillae vallatae.

\section{3) Die Zahl der Papillae vallatae.}

Zur Berechnung der Papillenzahl gibt es zwei Methoden : Die eine geht bei der Zählung von der Zahl der Umwallungen aus, es können dabei mehrere Papillen von einem Wall umschlossen sein; die andere Methode bestimmt die Zahl der Papillen selbst, wenn auch mehrere von einem Wall umgeben sind. Um die Zahl der Papillae vallatae genauer zu veranschaulichen, gebe ich beider Resultate an (nach Kunitomo). In meiner Tabelle 2, 6, 8 und 9 zeigen die eingeklammerten Ziffern die Befunde bei der Zählung der Umwallungen.

Tabelle 6. Zahl der lateralen Papillen an den einzelnen Zungen bei den Zwillingsfeten.

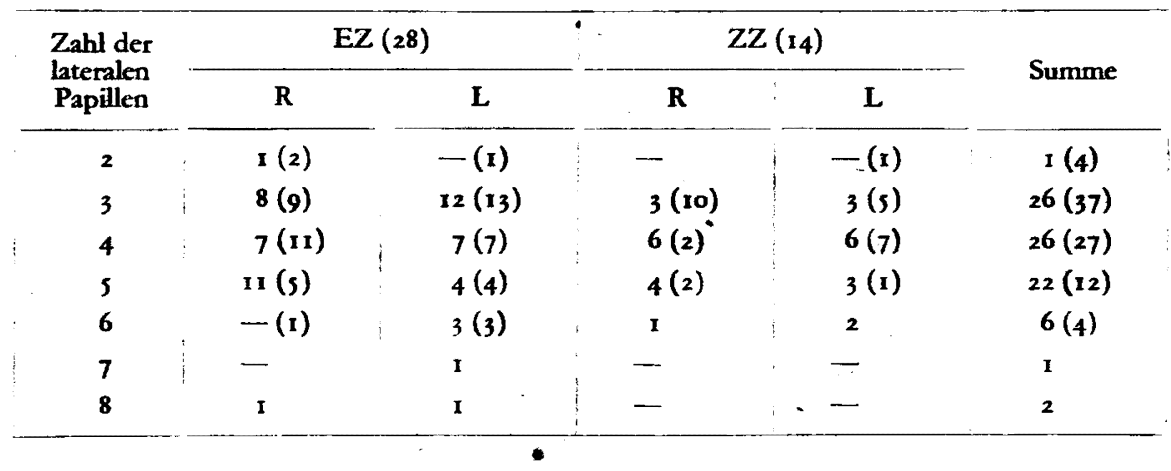

Wie man aus der Tabelle 6 ersieht, ist bei 28 EZ-Zungen die häufigste Zahl der Papillae vallatae, die zur einseitigen lateralen Papillenreihe gehören, 3 und bei ${ }_{4}$ ZZ-Zungen dieselbe 4. Wenn man aber eine von einem Wall umgebene Papillengruppe als eine Papille annimmt, dann ist die häufigste Zahl sowohl bei $\mathrm{EZ}$ als auch bei $\mathrm{ZZ} 3$.

Bei 216 Japanern von I-90 Jahren, nach Mochizuki, ist die häufigste Zahl der Papillae vallatae, die zur einseitigen lateralen Papillenreihe gehören, 4.

Die Zahl der lateralen Papillen ist bei den Zwillingsfeten in II von 28 EZ-Feten und in 7 von 14 ZZ-Feten, also insgesamt in 18 von 42 Feten beiderseitig gleich.

Nach Mochizuki ist die Zahl der lateralen Papillen in 81 unter 216 
Japanern ron $1-90$ Jahren auf den beiden Zungenseiten desselbe Individuums gleich. Er gibt in seiner Untersuchung an, dass die Zahl der lateralen Papillen bei den Japanern keinen bedeutenden Unterschied nach dem Geschlecht wie auch nach der Körperseite zeigt.

Nun werde ich auf die Zahl der einzelnen Papillengruppen bei den Zwillingsfeten etwas genauer eingehen und dann darüber die beiden Feten desselben Paares miteinander vergleichen.

Wie aus der Tabelle $7 \mathrm{zu}$ sehen ist, ist die häufigste Zahl der medianen Papillen sowohl bei EZ als auch bei $\mathrm{ZZ}$ I und die Hāufigkeit der Fälle, bei denen die mediane Gruppe aus 3 Papillen besteht, bei EZ auffallend grösser als bei $\mathrm{ZZ}$. Wir müssen aber die Tatsache in Betracht ziehen, dass in den Untersuchungsmaterialien die Zahl der älteren Feten bei EZ grösser als bei $\mathrm{ZZ}$ ist.

Tabelle 7. Zahl der medianen Papillen an den einzelnen Zungen bei den

Zwillingsfeten.

\begin{tabular}{cccc}
\hline $\begin{array}{c}\text { Zahl der } \\
\text { medianen } \\
\text { Papillen }\end{array}$ & \multicolumn{3}{c}{ Zahl der Zungen } \\
\cline { 2 - 4 } & EZ (28) & ZZ (14) & Summe \\
\cline { 1 - 4 } 1 & 3 & 1 & 4 \\
2 & 12 & 7 & 19 \\
3 & 6 & 5 & 12 \\
\hline
\end{tabular}

Beim II. Fetus eines EZ-Paares (Fall II), bei beiden Feten des anderen EZ-Paares (Fall 14) und beim je I. Fetus der zwei ZZ-Paare (Fall 16 und 19), also an s Zungen, wie die Tabelle 2 zeigt, befindet sich der mediane Wall, von dem zwei Papillen umschlossen werden.

Wie man aus der Tabelle 8 ersieht, ist die häufigste Zahl der sämtlichen Papillen bei EZ wie auch bei ZZ 9. Wenn man aber eine von einem Wall umgebene Papillengruppe als eine Papille annimmt, dann ist die häufigste Zahl sowohl bei EZ als auch bei ZZ 8. Dieses Ergebnis bezüglich der Zahl der sämtlichen Papillen bei den Zwillingsfeten stimmt mit dem bei den Kindern von Kunitomo überein.

Die Zahl der sämtlichen Papillen bei den Zwillingsfeten scheint keinen nennenswerten Unterschied nach dem Geschlecht und dem Fetalmonat zu zeigen; wie es bei der Angabe Mochizu ki's bei den Zungen der Japanern der Fall ist.

Num möchte ich auf die Vergleichung der Zahl der Papillen der beiden Feten desselben Paares eingehen, in erster Linie auf die Vergleichung der Zahl der lateralen Papillen.

Bei nur einem EZ-Paar ist die Zahl der lateralen Papillen an beiden Zungenhälften beider Feten 3 (vollkommene Konkordanz).

In 6 von 14 EZ-Paaren und in 2 von 7 ZZ-Paaren zeigt die Zahl der lateralen Papillen eine Konkordanz auf zwei gleichnamigen Zungenhälften der beiden Paarlinge desselben Paares, also die Häufigkeit der Kon- 
kordanz der Zahl der lateralen Papillen ist bei EZ etwas grösser als bei ZZ.

Bei EZ wie auch bei $Z Z$ in bezug auf die lateralen Papillen kommt keine Konkordanz der Zahl auf je zwei spiegelbildlich entgegengesetzten Zungenhälften vor.

Tabelle 8. Zahl der sämtlichen Papillen bei den Zwillingsfeten unter

Berücksichtigung des Vergleiches mit dem Ergebnis von Kunitomo.

\begin{tabular}{|c|c|c|c|c|}
\hline \multirow{3}{*}{$\begin{array}{l}\text { Zahl der } \\
\text { Papillen }\end{array}$} & \multicolumn{4}{|c|}{ Zahl der Materialien } \\
\hline & \multicolumn{3}{|c|}{$4^{2}$ Zwillingsfeten ( $\mathrm{g} a$ wa) } & \multirow{2}{*}{$\begin{array}{c}20 \text { Kinder } \\
(\mathrm{Kunitomo})\end{array}$} \\
\hline & $\mathrm{EZ}(28)$ & $Z Z(14)$ & Summe (42) & \\
\hline 5 & $-(\mathrm{x})$ & - & $-(\mathrm{I})$ & - \\
\hline 6 & $2(2)$ & $-(2)$ & $2(4)$ & $-(\mathrm{I})$ \\
\hline 7 & I (2) & $I(2)$ & $2(4)$ & $3(3)$ \\
\hline 8 & $5(8)$ & I (6) & $6(14)$ & $2(s)$ \\
\hline 9 & $7(4)$ & $6(1)$ & $I_{3}(5)$ & $5(3)$ \\
\hline 10 & $2(4)$ & 2 & $4(4)$ & $5(4)$ \\
\hline II & $s(s)$ & $I(3)$ & $6(8)$ & I (2) \\
\hline 12 & $3(x)$ & 2 & $s(\mathrm{I})$ & $x(x)$ \\
\hline 13 & $I(x)$ & - & $x(x)$ & $\mathbf{I}$ \\
\hline 14 & 2 & - & 2 & I ( 1$)$ \\
\hline Is & 一 & $\mathbf{x}$ & $\mathbf{r}$ & I \\
\hline
\end{tabular}

Wenn man eine von einem Wall umgebene Papillengruppe als eine Papille annimmt, dann ist die Konkordanz der Zahl der lateralen Papillen auf zwei gleichnamigen Zungenhälften der beiden Paarlinge in 7 mal von ${ }_{4}$ EZund in 3 von 7 ZZ-Paaren zu finden und bei einem ZZ-Paar. befindet sich eine vollkommene Konkordanz (beiderseits beider Feten dieses Paares 3 Papillen).

Zur Bezeichnung der Verschiedenheit der Zahl der beiderseitigen lateralen Wallpapillen der beiden Paarlinge desselben Zwillingspaares und weiter zur Erklärung des Unterschiedes zwischen $\mathrm{EZ}$ und $\mathrm{ZZ}$ wurde die prozentuale Abweichung nach v. Verschuer berechnet (Tabelle 9).

Bezüglich der mittleren prozentualen Abweichung für die Zahl der rechten lateralen Papillen der beiden Paarlingè findet sich kein merklicher

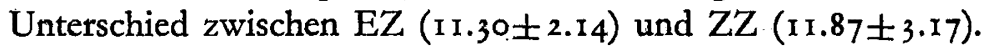

Die mittlere prozentuale Abweichung für die Zahl der linken lateralen Papillen der beiden Paarlinge ist bei EZ $13.85 \pm 2.62$ und bei ZZ $13.32 \pm$ 3.56.

Die mittlere prozentuale Abweichung für die Zahl der beiderseitigen 
Tabelle 9. Prozentuale Abweichung.

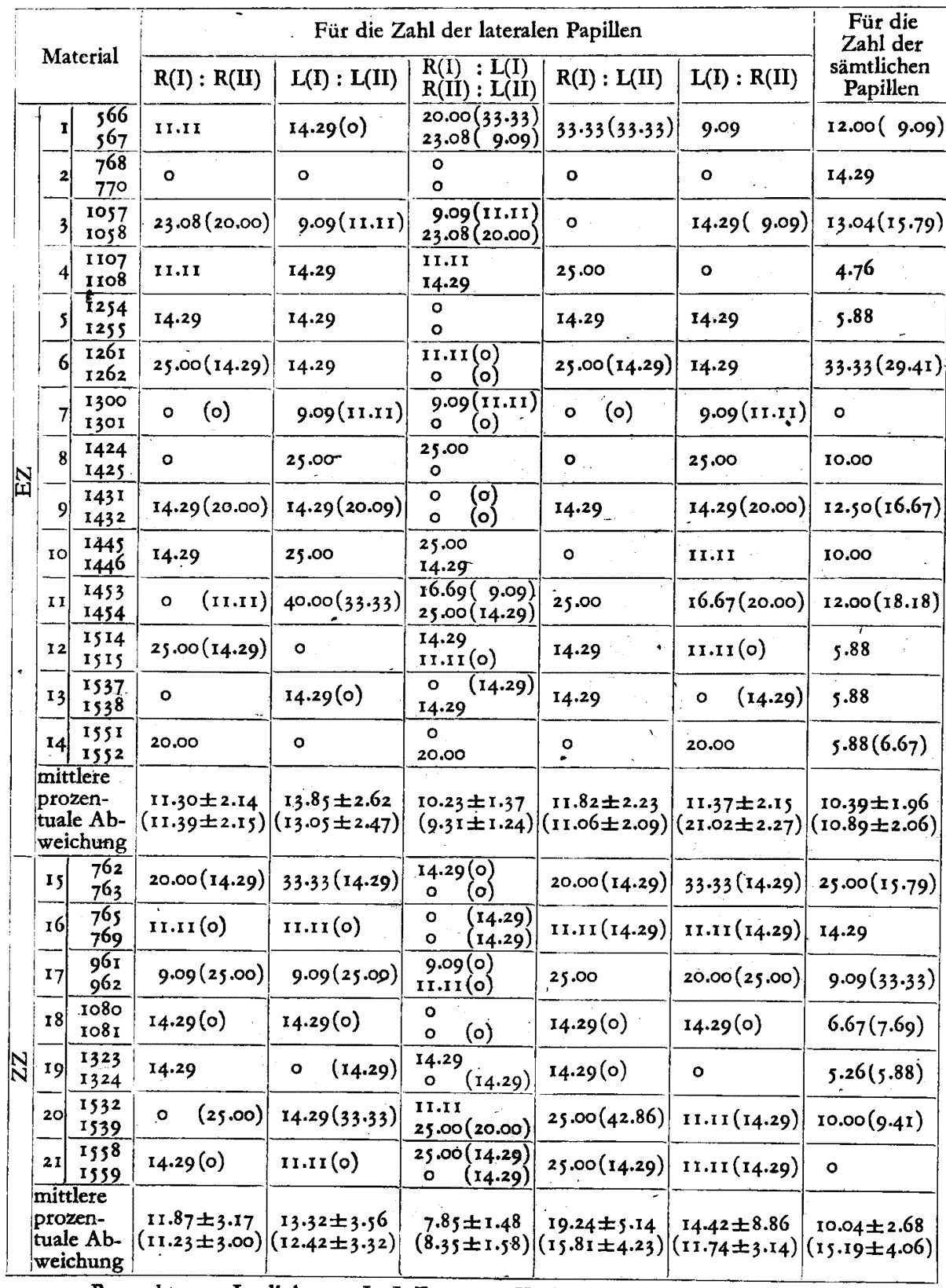

$$
R=\text { rechts, } \quad L=\text { links, } \quad I=I \text {. Fetus, } \quad I I=I I \text {. Fețus. }
$$

lateralen Papillen desselben Zwillingsindividuums beträgt bei EZ $10.23 \pm \mathrm{x} .37$ und bei $\mathrm{ZZ} 7.85 \pm 1.48$ 
Vergleichen wir die Zahl der rechten lateralen Papillen des I. Fetus und die der linken des II. Fetus desselben Paares miteinander, so ist die mittlere prozentuale Abweichung bei EZ I I.82 2.23 und bei $Z Z$ 19.24士5.14, daraus kann man nicht den Schluss ziehen, dass der Ähnlichkeitsgrad zwischen żwei Feten eines Paares bei EZ grösser als bei ZZ ist.

Die mittlere prozentuale Abweichung für die Zahl der linken lateralen Papillen des I. Fetus und der rechten des II. Fetus zeigt keinen merklichen Unterschied zwischen EZ (11.37士2.15) und ZZ (14.42士3.86).

Wenn man eine von einem Wall umgebene Papillengruppe als eine Papille berechnet, dann "zeigt die mittlere prozentuale Abweichung für die Zahł der lateralen Papillen auch keinen deutlichen Unterschied zwischen EZ und ZZ, wie die eingeklammerten Ziffern in der Tabelle 9 zeigen.

Was das Vorkommen der Doppelpapille anlangt, so besteht ein Konkordanz derart, dass die Doppelpapille in 3 von 14 EZ-Paaren an'beiden Zungenhälften beider Feten eines Paares und in 3 von 7 ZZ-Paaren an zwei gleichnamigen Zungenhälften beider Feten eines Paares vorkommt.

Die Zahl der medianen Papillen steht in 5 von 14 EZ- und in 3 von 7 ZZ-Paaren im könkordanten Verhältnis.

Um sich über die Verschiedenheit der Zahl der sämtlichen Papillae vallatae $\mathrm{zu}$ vergewissern, wurde die prozentuale Abweichung errechnet, die in der Tabelle 9 genau angegeben ist, woraus ich im folgenden die wichtigeren kurz mitteilen werde.

Die prozentuale Abweichung für die Zahl der sämtlichen Papillen zwischen zwei Feten ein und desselben Zwillingspaares beträgt beim I EZ- und I ZZ-Paar Null. Betreffs der mittleren prozentualen Abweichung kann man keinen deutlichen Unterschied zwischen EZ (10.39土 1.96) und ZZ (10.04士 2.68) bemerken. Wenn man eine von einem Wall umgebene Papillengruppe als eine Papille annimmt, dann beläuft sich die prozentuale Abweichung für die Zahl der Wallpapillen zwischen zwei Feten ein und desselben Zwillingspaares auch beim I EZ- und I ZZ-Paar auf Null. Die mittlere prozentuale Abweichung ist bei EZ $10.89 \pm 2.06$ und bei ZZ I 5.19 44.06 , daraus kann man nicht den Schluss ziehen, dass der Ähnlichkeitsgrad der Zahl der sämtlichen Papillen zwischen zwei Feten eines Paares bei EZ grösser als bei $\mathrm{ZZ}$ ist.

\section{v. Schluss.}

Der Winkel, welcher zwischen den beiderseitigen Schenkeln der lateralen Papillen zustandekommt, die Anordnung und die Zahl der Papillae vallatae bei den japanischen Zwillingsfeten wurden eingehend untersucht.; 
I. Das Untersuchungsmaterial betrifft insgesamt 2 I paarige Zwillinge (14 EZ-, 7 ZZ-Paare), d.h. 42 Feten (오 29, 우 13 ).

2. An 42 Zungen der Zwillingsfeten gehört die Stellung der Papillảe vallatae 22 mal zur V-, ig mal zur Y-und I mal zur T-Stellung. Die Häufigkeit der V- und Y-Stellung zeigt keinen bedeutenden Unterschied zwischen $\mathrm{EZ}$ und $\mathrm{ZZ}$ wie auch zwischen den männlichen Feten und den weiblichen.

3. Die Mehrzahl der Zungen zeigen bei den. Zwillingsfeten einen Winkel von $100^{\circ}-110^{\circ}$; häufig sind auch noch Zungen mit Winkeln von II $5^{\circ}$ und $120^{\circ}$. Dann nimmt die Zahl der Zungen nach beiden Seiten bis $92^{\circ}$ bzw. $155^{\circ} \mathrm{ab}$. Er zeigt keinen deutlichen Unterschied nach der Eiigkeit, dem Geschlecht und Fetalmonat.

4. Bei 28 EZ-Zungen bemerkt man als die häufigste Zahl der Papillae vallatae, die zur einseitigen lateralen Papillenreihe gehören, 3 und bei I 4 ZZ-Zungen als dieselbe 4 ; wenn man aber eine von einem Wall umgebene Papillengruppe als eine Papille annimmt, dann ist die häufigste Zahl sowohl bei $\mathrm{EZ}$ als auch bei $\mathrm{ZZ} 3$.

5. Die häufigste Zahl der sämtlichen Papillen ist bei EZ wie auch bei ZZ 9; wenn man aber eine von einem Wall umgebene Paṕillengruppe als eine Papille annimmt, dann ist die häufigste Zahl sowohl bei EZ als auch bei ZZ 8.

6. Was die Anordnung der Papillae vallatae und die Grösse des Winkels, welchen die beiderseitigen Schenkel der lateralen Papillen bildet, anlangt, so sind die Erbwirkungen auffallend nachzuweisen. Aber bezüglich der Zahl der lateralen, medianen oder sämtlichen Wallpapillen herrschen deutliche Umwelteinflusse.

Am Schlusse spreche ich meinen verehrten Lehrer, Herrn Prof. Dr. T. Taniguchi, für die stete freundliche Anregung und Unterstützung bei der Ausführung dieser Arbeit und für die Überlassung seines wertvollen Zwillingsmaterials meinen aufrichtigen Dank aus.

Mittel für diese Untersuchung wurden uns aus dem wissenschaftlichen Fonde des Unterrichtsministerium zur Verfügung gestellt, wofür wir hiermit unseren ergebensten Dank aussprechen. 


\section{Literatur.}

i) Grabert, W., Vergleichende Untersuchungen an Herero- und Hottentottenzungen. Archiv f. Anatomie und Physiologie. Anat. Abt. 1910.

2) Hopf, K. und Edzard, D., Beobachtungen über die Verteilung der Zungenpapillen bei verschiedenen Menschenrassen. Zeitsch. f. Morph. u. Anthrop. Bd. I2. I9ro.

3) Jurisch, A., Studien über Papillae vallatae beim Menschen. Zeitsch. f. Anatomie und Entwicklungsgeschichte. Bd. 66. 1922.

4) Kukita, S., Beiträge zur physischen Anthropologie der Ainu. 15. Mitteilung. Über die Papillae vallatae der Ainu. Fukuoka-Ikadaigaku-Zasshi. Vol. 25. 1932. (Japanisch).

5) Kunit omo, K., Über die Zungenpapille und die Zungengrösse der Japaner. Zeitsch. f. Morphologie und Anthropologie. Bd. 14. 1912.

6) Kunze, G., Die Zungenpapillen der Primaten. Morphologisches Jahrbuch. Bd.49. 1915.

7) Miyashita, K., Über die umwallte Zungenpapille der Chinesen. The Journal of Oriental Medicine. Vol. 22. I935. (Japanisch).

8) Mochizuki, Y., Studien über die umwallten Zungenpapillen der Japaner. Shika-Geppo. Bd. 18. 1938. (Japanisch).

9) —_, Ein neuer Typus der Stellung der.Papillae vallatae. Shika-Geppo. Bd. '19. 1939. (Japanisch).

ro) Nishi, K., Über die umwallte Zungenpapille der Japaner. Chiba-Igakkai-Zasshi. Bd. 5. 1927. (Japanisch).

Ir) Zuckermann, N., Beobachtungen über den Ventriculus laryngis und die Zungenpapillen einiger Melanesier. Zeitseh. f. Morph. u. Anth. Bd. I5. I913. 